\title{
Acompanhamento audiológico de lactente com risco para sífilis congênita: relato de caso
}

\author{
Audiological follow up of an infant affected by congenital syphilis: case report
}

\author{
Dayana da Silva Santos ${ }^{1}$, Maria Cecília Castello Silva Pereira ${ }^{2 *}$, Eduardo Pondé de Sena ${ }^{3}$
}

\begin{abstract}
1 Fisioterapeuta. Mestranda do Programa de Pós-Graduação em Processos Interativos de Órgãos e Sistemas, Instituto de Ciências da Saúde, Universidade Federal da Bahia; 2 Fonoaudióloga. Doutora em Processos Interativos de Órgãos e Sistemas, pela Universidade Federal da Bahia, docente do curso de Fonoaudiologia da Universidade do Estado da Bahia.; 3 Professor Associado do Departamento de Biorregulação e do Programa de Pós-graduação em

Processos Interativos de Órgãos e Sistemas, Instituto de Ciências da Saúde, Universidade Federal da Bahia.
\end{abstract}

\begin{abstract}
Resumo
Objetivo: descrever o acompanhamento audiológico de uma criança com exame sorológico positivo para sífilis. Caso Clínico: E.G.M.L, com sorologia positiva para sífilis, com dois dias de vida, obteve resultado "falha" na Triagem Auditiva Neonatal, com Emissões Otoacústicas Evocadas Transientes. Encaminada para o ambulatório da Clínica Escola de Fonoaudiologia, da Universidade Estadual da Bahia, retornou com quatro meses, quando realizou reteste das Emissões Otoacústicas Evocadas Transientes, que permaneceram ausentes; também se submeteu à imitanciometria, onde foram obtidas curvas timpanométricas "pico único". A avaliação da condução nervosa com Potencial Evocado Auditivo do Tronco Encefálico do Tronco Encefálico mostrou-se normal na orelha esquerda, com limiares eletrofisiológicos presentes até a intensidade de $50 \mathrm{~dB}$. Porém, houve despertar do sono e não foi possível avaliar a orelha direita. Em nova reavaliação, aos oito meses, a condução nervosa pelo mesmo processo apresentou normalidade na orelha direita. As condições nutricionais de E.G.M.L. eram então críticas, com desnutrição acentuada. $O$ atendimento no âmbito audiológico foi temporariamente suspenso e houve encaminhamento para o setor de fisioterapia e nutrição da Universidade do Estado da Bahia. Discussão: o levantamento de questões audiológicas, em grupos de risco para alterações auditivas, visa à reabilitação e à garantia das condições ideais de comunicação. Neonatos com detecção precoce de alterações auditivas são candidatos ideais à amplificação e reabilitação. Conclusão: crianças com risco para sífilis congênita precisam ser avaliadas na Triagem Auditiva e acompanhadas no seguimento. Entretanto, algumas vezes torna-se difícil conscientizar a família. Essa continuidade é importante para assegurar a integridade dos sentidos e, no caso da audição, para favorecer o desenvolvimento adequado da criança.
\end{abstract}

Palvras-chave: Infecção congênita. Lactente. Triagem auditiva neonatal.

\begin{abstract}
Objective: to describe the audiologic follow-up of a child testing positive in the serological test for syphilis. Case: E.G.M.L. has positive syphilis serology and within two days of life presented a Newborn Hearing Screening result considered fail and Transient-evoked Otoacoustic Emissions (TEOAE). She was referred to the outpatient clinic of the Clinical School of Speech-Language Therapy of State University of Bahia (UNEB) to where she returned after four months to submit to a retest of TEOAE which remained absent and an immittance test that presented a single-peaked tympanometric shape. The evaluation of nerve conduction related to Auditory Brainstem Response ( $A B R$ ) was normal in the left ear presenting thresholds up to $50 \mathrm{~dB}$. However, as she awakened, it was not possible to assess the right ear. However, in a new reassessment, which only occurred when she was at eight months of age, the nerve conduction related to $A B R$ was normal in the right ear. The nutritional status of E.G.M.Lat eight months of age was critical, she was severely undernourished. The hearing care service was temporarily suspended, then she was referred to the UNEB physiotherapy and nutrition sector. Discussion: the purpose of the survey on audiological issues among infectious risk groups is to make an early diagnosis of hearing disorders in order to promote rehabilitation and improve communication functions. Neonates with hearing loss at such a young age are the best candidates for amplification and rehabilitation. Conclusion: Children at risk for congenital syphilis need to be evaluated in terms of Hearing Screening and to be monitored, even though it sometimes becomes difficult to convince the family about that. It is important to keep this process in order to assure the integrity of the senses, especially in terms of the audiologic system, and to promote the adequate development of the child.
\end{abstract}

Keywords: Congenital infection. Infant. Newborn hearing screening.

\section{INTRODUÇÃO}

A surdez incide em 1 a 3 a cada mil nascimentos e a cada 2 a 10 por mil nascimentos de neonatos de alto risco.

Correspondente/Corresponding: *Maria Cecília Castello Silva Pereira - End: Rua Silveira Martins, 2555 - Cabula. CEP: 41150-000 - E-mail: mcpereira@uneb.br; cecília.castello@hotmail.com
Quanto à etiologia, pode ser de origem genética, idiopática e de causas perinatais, dentre as quais se destacam as infecções congênitas e, em especial, a sífilis (AMERICAN ACADEMICS PEDIATRICS, 2019).

Aproximadamente 1,5 milhões de mulheres grávidas estão infectadas com sífilis ativa e cerca de metade delas não é tratada e poderá ter sequelas, como perda fetal pre- 
coce, morte neonatal, bebês com baixo peso ao nascer e lactentes com evidências clínicas de infecção. A prevenção da transmissão vertical, da mãe para o filho, comumente referida como sífilis congênita, é relativamente simples e de baixo custo. A transmissão da mãe para o bebê pode ser evitada quando o tratamento na fase pré-natal é adequado (ORGANIZAÇÃO MUNDIAL DA SAÚDE, 2014).

No Brasil, de 2005 a junho de 2016, foram notificados 169.546 casos de sífilis em gestantes, no Sistema de Informação de Agravos de Notificação (SINAN), sendo 21,7\% na região Nordeste.

A sífilis congênita (SC) é uma doença de notificação compulsória, decorrente da disseminação hematogênica do Treponema pallidum (T. pallidum) por via transplacentária, da gestante não tratada ou inadequadamente tratada para o seu concepto (COOPER et al, 2016; SÃO PAULO. SECRETARIA DE ESTADO DE SAÚDE, 2016; SILVEIRA, 2017).

Quanto à enfermidade em pauta, em 2019, foram registrados 24.130 casos no país. Já, de acordo com o Boletim Epidemiológico de Sífilis, em 2019 foram notificados 152.915 casos de sífilis adquirida em todo o país, com taxa de detecção de 72,8 casos por 100 mil habitantes. Nos últimos anos, houve um progressivo aumento na taxa de incidência de sífilis congênita: em 2009, a taxa era de 2,1 casos/1.000 nascidos vivos e em 2018 chegou a 9,0 casos/1.000 nascidos vivos, reduzindo para 8,2 casos $/ 1.000$ nascidos vivos em 2019. A maior parte das notificações ocorreu em indivíduos entre 20 e 29 anos (36,2\%). (BRASIL. MINISTÉRIO DA SAÚDE, 2006)

Em relação à detecção nacional de casos, houve uma redução de $4,5 \%$ na taxa quando comparada a 2018 , que apresentou taxa de 76,2 por 100 mil habitantes. Em gestantes, foram 61.127 casos em 2019, com redução de 3,3\% em relação ao ano anterior que, por sua vez, foi de 63.182 casos (BRASIL. MINISTÉRIO DA SAÚDE, 2020). A região Nordeste ocupa o segundo lugar no ranking das regiões com maior número de casos de sífilis em gestantes no país, sendo o estado do Maranhão o que apresenta a maior incidência dessa região, com seis casos por mil nascidos vivos (CONCEIÇÃO; CÂMARA; PEREIRA, 2019). Na Bahia, foram registrados 635 casos de sífilis congênita, sendo 272 em Salvador, no ano de 2018 (BRASIL. MINISTÉRIO DA SAÚDE, 2020)

Quanto aos óbitos, em 2019 houve 173 notificações por sífilis congênita (em menores de um ano). No Brasil, nos últimos 10 anos, ocorreu aumento no coeficiente de mortalidade infantil por sífilis, que passou de 2,2 por 100 mil nascidos vivos em 2009, para 5,9 por 100 mil nascidos vivos em 2019. Em 2018, o coeficiente de mortalidade infantil por sífilis foi de 8,9 por 100 mil nascidos vivos (BRASIL. Ministério da Saúde, 2020)

A taxa de transmissão vertical da sífilis, nas fases primárias e secundárias da doença, é de $70 \%$ a $100 \%$ nas gestantes que não recebem tratamento e/ou são tratadas inadequadamente, com redução nas fases latente e tardia. A infecção pela T. pallidum pode desencadear aborto espontâneo, malformações congênitas, natimorte ou morte perinatal em cerca de $40 \%$ das crianças infectadas (BRASIL. Ministério da Saúde, 2006; CONCEIÇÃO; CÂMARA; PEREIRA, 2019).

Os resultados da sífilis congênita incluem edema fetal não imune, icterícia, hepatoesplenomegalia, rinite, erupção cutânea, pseudoparalisia e surdez (TORRES et al., 2019).

As infecções na gestação representam uma importante causa de perda auditiva neurossensorial. A perda auditiva causada pela sífilis congênita varia de 25 a 38\%, podendo ser flutuante e acompanhada ou não de sinais vestibulares (CHAU et al., 2009).

As manifestações da doença podem estar ausentes no nascimento e surgir tardiamente, mas também de forma precoce (até o segundo ano de vida) ou tardia (após o segundo ano de vida), sendo os desfechos para a infecção congênita; assistência pré-natal inadequada, tratamento insuficiente ou inadequado para sífilis, além da prematuridade e baixo peso ao nascer estão associados ao diagnóstico laboratorial positivo para sífilis em recém-nascidos (TORRES et al., 2019).

$O$ baixo peso ao nascer (BPN) é definido pela Organização Mundial da Saúde como inferior a 2,5kg (WORLD HEALTH ORGANIZATION, 2014), está associada à mortalidade neonatal (MOREIRA; SOUSA; SARNO, 2018) e relacionada ao processo de desnutrição.

A desnutrição corresponde a uma doença de natureza clínico-social multifatorial, cujas raízes se encontram na pobreza. Quando ocorre na primeira infância, está associada à maior mortalidade, à recorrência de doenças infecciosas, a prejuízos no desenvolvimento psicomotor, ao menor aproveitamento escolar e à menor capacidade produtiva na idade adulta. A desnutrição é considerada um importante problema de saúde pública, nessa faixa etária, nos países em desenvolvimento, a exemplo do Brasil (MONTEIRO et al., 2009). Ela é frequentemente descrita por termos de índices antropométricos, comparando peso e altura com as normas populacionais, e constitui-se a base para a identificação precoce de deficiências nutricionais, podendo estar aliada à presença de doenças (CARAM; MORCILLO, COSTA PINTO, 2010; WORLD HEALTH ORGANIZATION, 2008).

Foram levantados nos prontuários de uma maternidade pública dados de neonatos com diagnóstico de sífilis, detectados ao nascimento através de exames sorológicos. Em seguida, os responsáveis pelos neonatos foram contatados e convidados a participar da pesquisa para avaliar a audição dos pesquisados, após aprovação pelo Comitê de Ética em Pesquisa da Universidade Estadual da Bahia (UNEB), sob o número de protocolo 1.396.094.

A lista de pacientes acometidos por sífilis congênita, comprovados pelo Venereal Disease Research Laboratory (VDRL), no período de dezembro de 2015 a outubro de 2016, foi de 110 mães. OVDRL é um tipo de Teste não treponêmico, usado para triagem da sífilis. (TORRES et al., 2019).

O objetivo do presente relato é descrever o acompanhamento audiológico de uma criança com exame sorológico positivo para sífilis. 


\section{CASO CLÍNICO}

E.G.M.L, filha de R.G.S, última filha de um total de nove irmãos, nasceu em janeiro de 2016, na Maternidade José Maria de Magalhães Neto.

Com dois dias de vida, foi realizada a Triagem Auditiva Neonatal com Emissões Otoacústicas Evocadas Transientes, com aparelho OtoRead (Interacoustics), quando se observou falha bilateral. Nos dados do prontuário, constam: idade gestacional 38 semanas e 2 dias (a termo - RNT), peso ao nascimento $1.805 \mathrm{~g}$ (baixo peso-BP), Apgar 9 (1 min) e 10 (5 min), crescimento intrauterino (CIUR) desproporcional. Agenitora não realizou pré-natal. Apresentou líquido amniótico (LA) fétido, bolsa rota $>18$ horas o que caracterizou risco infeccioso. Foi um neonato considerado pequeno para a idade gestacional (PIG). $O$ exame VDRL foi positivo, contagem da mãe 1/1 e RN 1/1, portanto com risco para TORCHS (sífilis congênita).

Devido à sorologia positiva para sífilis, E.G.M.L usou penicilina procaína por oito dias $(D=8)$. No dia da alta, fez uso de penicilina benzatina.

Realizou avaliação do líquor, com resultados negativos para neurossífilis. Recebeu alta no dia 14 de janeiro de 2016. No dia da alta, foi realizado reteste das EOAT, permanecendo falha bilateral.

E.G.M.L foi encaminhada para o ambulatório da Clínica Escola de Fonoaudiologia da UNEB para realização de Potencial Evocado Auditivo do Tronco Encefálico (PEATE) e reteste das EOAT.

Deveria retornar com um mês, mas só retornou com quatro meses, quando realizou reteste das Emissões Otoacústicas Evocadas Transientes e Imitanciometria, além de PEATE.

As EOAT mantiveram-se ausentes. Como mostrado na
Figura 1, na imitanciometria, foram obtidas curvas timpanométricas "pico único", caracterizando normalidade nas condições de orelha média, com reflexos acústicos estapedianos ausentes na via aferente ipsilateral esquerda (f $1 \mathrm{k}$ e $2 \mathrm{kHz}$ ) e direita $(2 \mathrm{kHz})$, somente presentes na via ipsilateral direita na $\mathrm{f} 1 \mathrm{kHz}$

Figura 1-Análise das curvas timpanométricas nas duas orelhas com o equipamento Otoflex (Otometrics)

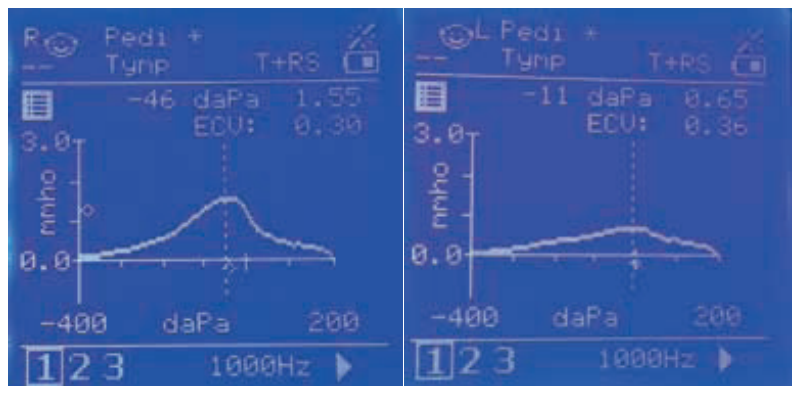

Curva timpanométrica $O D^{*}$

Curva timpanométrica $O E^{*}$

Fonte: Autoria própria

Nesta ocasião, a avaliação da condução nervosa com PEATE mostrou-se normal na orelha esquerda e os limiares eletrofisiológicos foram presentes até a intensidade de 50 dB (Figura 2). Com relação à orelha direita, em função do despertar do sono da criança, não foi possível realizar a avaliação. Solicitou-se à mãe um retorno em 15 dias para continuação do PEATE. Uma nova reavaliação só ocorreu aos oito meses. A condução nervosa no PEATE apresentou normalidade na orelha direita (Figura 3).

Figura 2 - Gráfico PEATE realizado em maio de 2016 na orelha esquerda com o Equipamento Contronics - ATC Plus

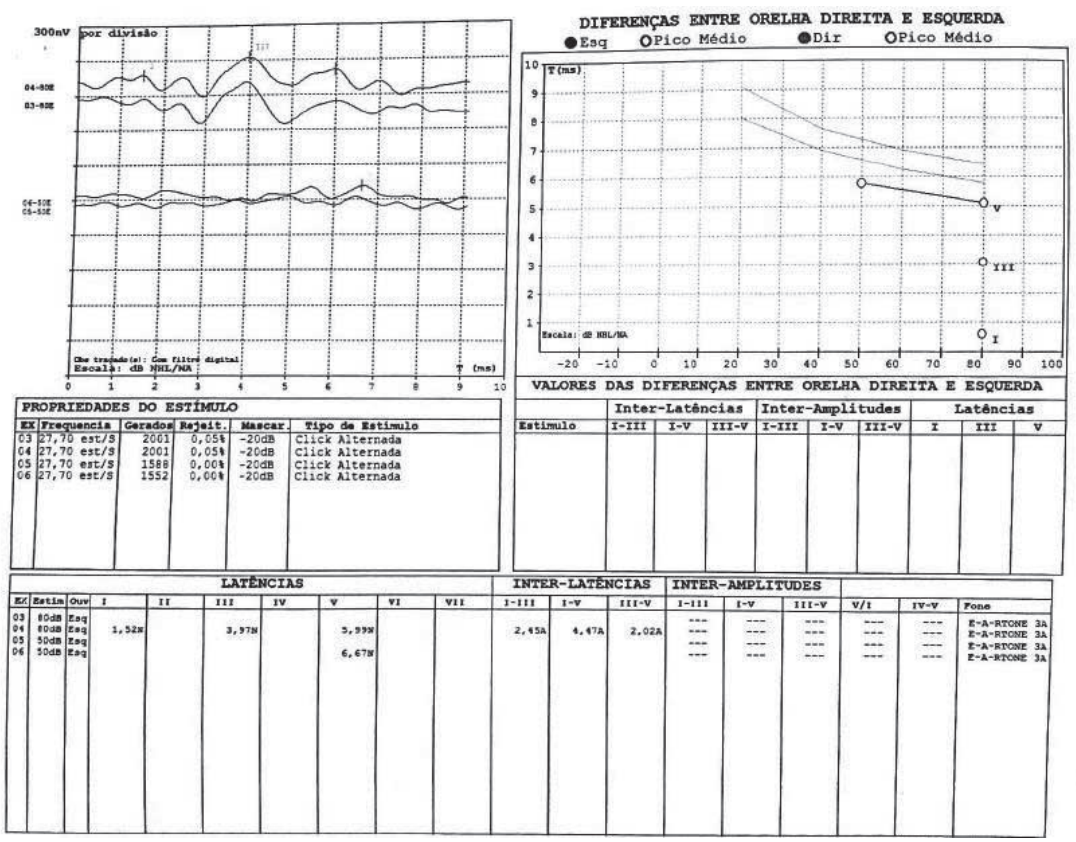


Fonte: Dados da pesquisa.

Figura 3 - Gráfico PEATE realizado em setembro de 2016 na orel ha direita com o Equipamento Contronics - ATC Plus.

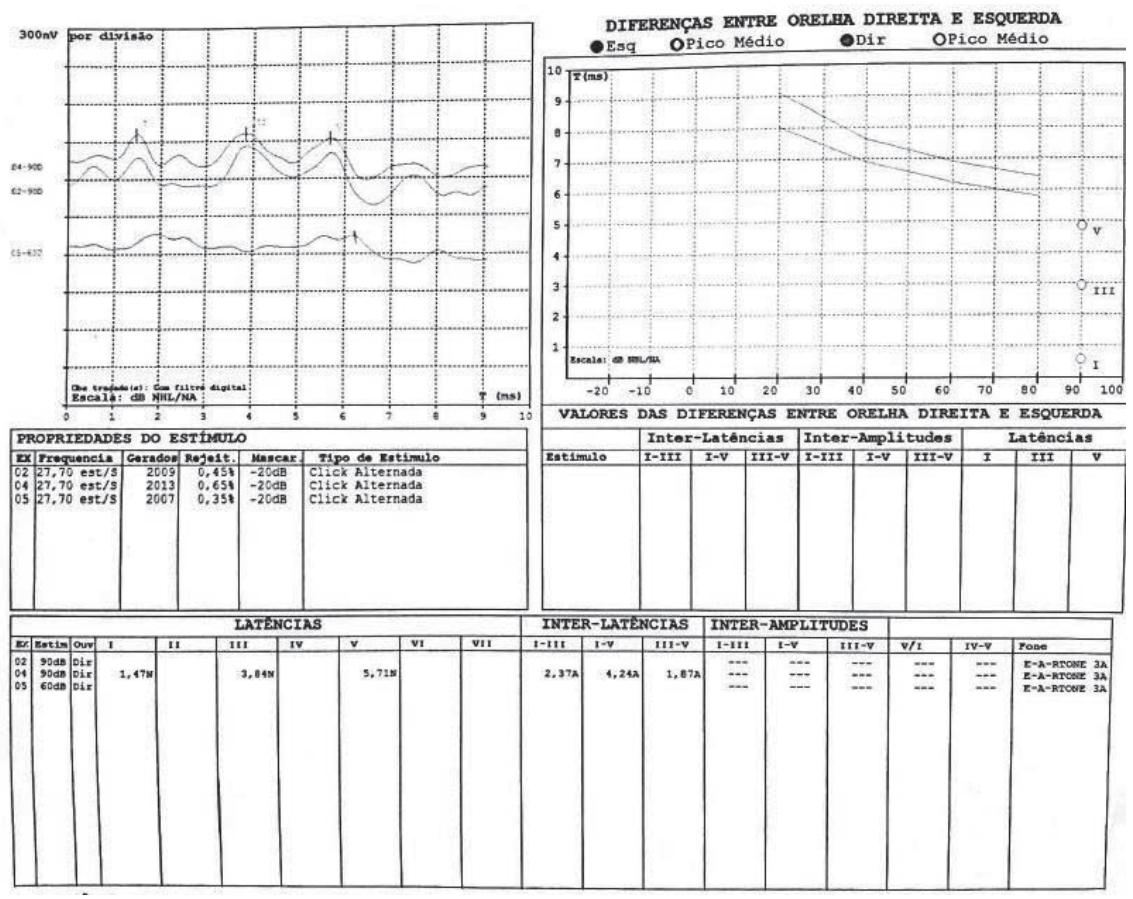

Fonte: Dados da pesquisa.

Neste segundo retorno, as condições nutricionais de E.G.M.L aos oito meses eram muito críticas, com desnutrição acentuada. Ante o fato, o acompanhamento no âmbito audiológico foi interrompido e a criança foi encaminhada ao setor de Fisioterapia e Nutrição da Clínica Escola da UNEB.

\section{DISCUSSÃO}

As alterações audiológicas encontradas indicaram uma possível perda auditiva sensorioneural, com emissões otoacústicas ausentes e PEATE alterado, embora incompleto. 0 retorno para reteste e fechamento do quadro audiológico não foi concluído, devido ao tempo entre os retornos para reteste e as demandas que ocorreram. Alguns estudos demonstram as dificuldades no retorno e finalização do diagnóstico. (PINTO et al., 2019).

Nos casos em que ocorre falha nas emissões otoacústicas, a imitanciometria de altas frequências é uma importante ferramenta no diagnóstico diferencial da audição, já que descarta uma alteração de orelha média (JACOB-CORTELETTI et al., 2015; TATINAZZIO et al., 2011).

A SC é responsável pelo nascimento de bebês com restrição de crescimento em até $40 \%$ dos casos, levando a baixo peso, como o caso apresentado. (ALBUQUERQUE et al., 2014; MINISTÉRIO DA SAÚDE, 2012).

Fatores como desnutrição intrauterina e pós-natal, partos prematuros, rápido abandono do leite materno, doenças, infecções repetidas, ingestão insuficiente de alimentos capazes de suprir as necessidades da criança, tanto de energia quanto de proteína, falta de conhecimen- tos básicos sobre higiene, desemprego, proles numerosas e o vínculo mãe/filho enfraquecido também podem ser considerados como relevantes na origem da desnutrição (FRAGA; VARELA, 2014).

A desnutrição é um problema que atinge principalmente as crianças e que pode causar sérios danos ao seu desenvolvimento, como debilidade imunológica, retardo do crescimento, comprometimento do desenvolvimento intelectual, psicomotor e cerebral, entre muitas outras situações de risco (FRAGA; VARELA, 2014).

Frente às precárias condições nutricionais apresentadas por E.G.M.L, aos oito meses, além da surdez diagnosticada, houve uma preocupação referente ao processo evolutivo no seu ambiente social e as possíveis repercussões que poderiam impactar na sua qualidade de vida, pois, segundo Fraga e Varela (2014), alterações do desenvolvimento em uma criança, em função de um processo desnutrição, podem produzir efeitos significativos nesses dois aspectos indicados. Diante disto, nota-se a importância de uma abordagem interdisciplinar que inclua os seguintes profissionais: o médico, para assistência e controle patológico; o fisioterapeuta, para acompanhamento e adequação do desenvolvimento neuropsicomotor; o nutricionista, para controle nutricional; e o fonoaudiólogo, para acompanhamento audiológico diante de risco de surdez.

No presente caso, apesar da experiência com gestações anteriores, não houve acompanhamento pré-natal. Estudos mostram correlação entre acompanhamento pré-natal e peso ao nascer, com risco auditivo e de linguagem (NASCIMENTO et al., 2020). 
Malformações congênitas, doenças hereditárias e infecções como a SC, que acometeram E.G.M.L, entre outras situações adversas, podem ser um risco para o desenvolvimento normal da criança. Passando essa fase, outros riscos aparecem no período peri e pós-natal. Esses riscos são mais perceptíveis na infância porque os primeiros anos são marcados por importantes formações motoras, sociais, físicas e mentais. É também o período em que a criança está muito suscetível aos estímulos do meio (SACCANI et al., 2007). Como não foi possível dar continuidade ao acompanhamento da criança e diante das problemáticas descritas e apresentadas por ela, acreditamos que o seu crescimento tenha ocorrido com um atraso no desenvolvimento neuropsicomotor e possíveis alterações comportamentais.

\section{CONCLUSÃO}

Assim, entendemos que um dos maiores desafios nas alterações sensoriais é conscientizar a família sobre a importância da integridade dos sentidos, no caso a audição, para o desenvolvimento adequado de fala e linguagem. Neste processo, porém, condições primárias, como a nutrição e o ganho de peso, tornam-se prioritários para que se dê continuidade ao atendimento. A reabilitação deve ocorrer com a criança em equilíbrio no seu desenvolvimento global. Neste aspecto, a família é primordial, para que perdas de seguimento não ocorram.

\section{REFERÊNCIAS}

AMERICAN ACADEMY OF PEDIATRICS. Supplement to the JCIH 2019 Position Statement: Principles and guidelines for early hearing detection and intervention programs. Pediatrics, Evanston, v. 4, n. 2, p. 1-44, 2019.

BRASIL. Ministério da Saúde. Brasil avança no enfrentamento à sífilis. Brasília, 29 out. 2020. Disponível em: http://www.aids.gov.br/pt-br/ noticias/brasil-avanca-no- enfrentamento-sifilis. Acesso em: 19 nov. 2020.

BRASIL. Ministério da Saúde. Secretaria de Vigilância em Saúde. Programa Nacional de DST/AIDS. Diretrizes para controle da sífilis congênita: manual de bolso. 2. ed. Brasília, 2006. 72 p. (Série Manuais, 24).

CARAM, A.L.; MORCILLO, A.M.; COSTA PINTO, E. A. da. Nutritional status of children with cerebral palsy. Rev nutr., Campinas, v. 23, n. 1, p. 211219, 2010

$\mathrm{CHAU}$, J. et al. A systematic review of pediatric sensorineural hearing loss in congenital syphilis. Int. j. pediatr. otorhinolaryngol.. Amsterdam, v. 73, n. 6, p. 787-792, 2009.

CONCEIÇÃO, H. N. da; CÂMARA, J. T.; PEREIRA, B M. Análise epidemiológica e espacial dos casos de sífilis gestacional e congênita. Saúde em Debate, Londrina, v. 43, n.123, p. 1145-1158, 2019. Disponível em: https://doi. org/10.1590/0103-1104201912313. Acesso em: 22 nov. 2020. DOI: 10.1590/0103-1104201912313.

COOPER, J.M. et al. In time: the persistence of congenital syphilis in Brazil - More progress needed. Rev. Paul. Pediatr., São Paulo, v. 34, p. 251-243, 2006.

FRAGA J. A. A.; VARELA, D. S. da S. A relação entre a desnutrição e o desenvolvimento infantil. Rev. Assoc. Bras. Nutr., São Paulo, v. 4, n. 1, p. 59-62, maio 2014.
JACOB-CORTELETTI, L.C.B. et al. A influência da sonda de 226 e $1.000 \mathrm{~Hz}$ no registro do reflexo acústico em neonatos. CoDAS, São Paulo, v. 27, n. 3, p. 223-229, 2015.

LAGO, E.G.; VACCARI, A.; FIORI, R.M. Clinical features and follow-up of congenital syphilis. Sex. transm. dis., Philadelphia, n. 40, p. 85-94, 2013.

MONTEIRO, C.A. et al. Causas do declínio da desnutrição infantil no Brasil, 1996-2007. Rev. Saúde Pública, São Paulo, v. 43, n. 1, p. 35-43, 2009. Disponívle em: http://www.scielo.br/scielo.php?script=sci_ arttext\&pid=S0034- $89102009000100005 \&$ lng=en\&nrm=iso.Acesso em: 28 nov. 2020. DOI: 10.1590/S0034-89102009000100005.

MOREIRA, A. I. M.; SOUSA, P. R. M.; SARNO, F. Baixo peso ao nascer e seus fatores associados. Einstein (São Paulo), São Paulo, v. 16, n. 4, eAO4251, 2018. Disponível em: http://www.scielo.br/scielo.php?script=sci arttext\&pid=S1679- 45082018000400202\&lng=en\&nrm=iso. Acesso em: 28 nov. 2020. DOI: 10.31744/einstein_journal/2018ao4251.

NASCIMENTO, G. B. et al. Indicadores de risco para a deficiência auditiva e aquisição da linguagem e sua relação com variáveis socioeconômicas, demográficas e obstétricas em bebês pré-termo e a termo. CoDAS, São Paulo, v. 32, n. 1, p. 1-9, 2020.

ORGANIZAÇÃO MUNDIAL DE SAÚDE. Surdez e perda auditiva. Pediatr. otorhinolaryngol., Amsterdam, v. 73, n. 6 p. 87-92, 2014. Disponível em: em: http://www.searo.who.int/thailand/factsheets/fs0003/en/. Acesso em: 27 nov. 2020.

PINTO, J.D. et al. Evasão na Triagem Auditiva Neonatal: relação com indicadores de risco para deficiência auditiva. Rev. CEFAC, São Paulo, v. 21. n. 4, p. 1-7, 2019.

SACCANI, R. et al. Avaliação do desenvolvimento neuropsicomotor em crianças de um bairro da periferia de Porto Alegre. Sci. Med., Australia, v. 3, n. 17, p. 130-137, 2007.

SÃO PAULO. Secretaria de Estado da Saúde. Coordenadoria de Controle de Doenças. Programa Estadual de DST e Aids. Centro de Referência e Treinamento em DST e Aids.

Guia de bolso para manejo da sífilis em gestantes e sífilis congênita. 2 . ed. São Paulo, 2016. Disponível em: ttps://issuu.com/crtdstaidsspcrtaids/ docs/guia_de_bolso_da_s_filis_-_2 2020. edi. Acesso em: 20 nov.

SILVEIRA, S.L. Estudo epidemiológico da sífilis congênita: a realidade de um hospital universitário terciário. 2017. 54f. Dissertação (Mestrado Profissional em Medicina) - Universidade Estadual Paulista, Botucatu, SP, 2017.

TATINAZZIO, T.G. et al. Emissões otoacústicas e medidas de imitância acústica com tons de sonda de 226 e $1000 \mathrm{~Hz}$ em lactentes. Rev. Cefac, São Paulo, v. 13, n. 3, p. 478-488, 2011.

TORRES, R. G. et al. Pregnancy: the reality in a public hospital. Rev. bras. ginecol. obstet.

Rio de Janeiro, v. 41, n. 2, p. 90-96, Feb. 2019. Disponível em: https://doi. org/10.1055/s-0038- 1676569. Acesso em: 28 nov. 2020.

WORLD HEALTH ORGANIZATION. 10 facts on nutrition. Geneva: WHO, 2008. Disponivel em: http://www.who.int/featuresL/factfiles/nutrition/e/ index.html. Acesso em: 20 nov. 2020.

WORLD HEALTH ORGANIZATION. Global nutrition targets 2025: low birth weight policy brief. Geneva: WHO, 2014. Disponível em: http://apps. who.int/iris/bitstream/10665/149020/2/WHO_NMH_NHD_14.5_eng. pdf?ua=1. Acesso em: 25 nov. 2020.

Submetido em: $07 / 12 / 2020$

Acesso em: $14 / 12 / 2020$ 